УДК 681.518

\title{
ЭНТРОПИЙНЫЙ ПОДХОД К ИССЛЕДОВАНИЮ ИНФОРМАЦИОННЫХ ВОЗМОЖНОСТЕЙ АДАПТИВНОЙ РАДИОТЕХНИЧЕСКОЙ СИСТЕМЫ ПРИ ВНУТРИСИСТЕМНОЙ НЕОПРЕДЕЛЕННОСТИ
}

\author{
СКАЧКОВ В. В., ЧЕПКИЙ В. В., БРАТЧЕНКО Г. Д., ЕФИМЧИКОВ А. Н. \\ Одесская государственная академия технического регулирования и качества, \\ Украина, Одесса, 65020, ул. Кузнечная 15
}

\begin{abstract}
Аннотация. Рассмотрена энтропийная метрика по Шеннону, модифицированная к решению задачи оценивания информационных возможностей адаптивной радиотехнической системы в условиях внутрисистемной неопределенности. Показано приложение энтропийного подхода как инструмента обобщенного представления известных критериев адаптивной обработки сигналов при внутрисистемных возмущениях параметрического вектора системы
\end{abstract}

Ключевые слова: энтропийный подход; информация; адаптивная радиотехническая система; внутрисистемные возмущения; внутрисистемная неопределенность; оценка информационных возможностей; вариационно-параметрический метод

\section{ПОСТАНОВКА ПРОБЛЕМЫ. АНАЛИЗ ИССЛЕДОВАНИЙ И ПУБЛИКАЦИЙ}

Задача исследования информационных возможностей адаптивных радиотехнических систем (РТС), выполняющих функции на основе использования информации о событиях, ситуациях и процессах, происходящих вне рассматриваемых систем либо в самих системах, относится к классу задач с априорной неопределенностью. Априорная неопределенность радиотехнической системы [1-4] порождается состоянием внешней среды ее пребывания (внешнесистемная неопределенность), и существованием источников случайных внутрисистемных возмущений (внутрисистемная неопределенность).

В классическом представлении характер внешнесистемной неопределенности зависит от класса решаемых задач, динамики изменения внешних условий, вида априорной инфор- мации об измеряемых и неизмеряемых параметрах состояния среды. В этом контексте допустимо полагать, что уровень преодоления внешнесистемной неопределенности определяется возможностями адаптивной РТС, выбранной в целевой задаче в качестве объекта исследования $[1,2]$.

Предпосылками внутрисистемной неопределенности в адаптивных РТС являются ресурсные ограничения, связанные с существованием внутренних шумов системы, ограниченной точностью проводимых вычислений, неадекватностью прямых и обратных преобразований наблюдаемых реализаций, отсутствием изоморфизма в реальной системе и др. [4-7]. Свойственные любой реальной системе, такие ресурсные ограничения на физическом уровне проявляются в виде случайных внутрисистемных возмущений, которые не могут быть устранены в принципе. Указанные обстоятельства актуализируют проблему количе- 